Bioscientia Medicina: Journal of Biomedicine \&

Translational Research

Journal Homepage: www.bioscmed.com

\title{
Role of Fibroblast Growth Factor-23 in Coronary Slow Flow Phenomenon Pathogenesis
}

\section{Welly Oktaviandani ${ }^{1}$, Taufik Indrajaya ${ }^{2}$, Ali Ghanie ${ }^{2}$, Erwin Sukandi ${ }^{2}$}

${ }^{1}$ Subspecialized Residency Training, Cardiovascular Division, Department of Internal Medicine, Faculty of Medicine, Universitas Sriwijaya/ Dr Moh Hoesin General Hospital, Palembang, Indonesia

${ }^{2}$ Medical Staff, Cardiovascular Division, Department of Internal Medicine, Universitas Sriwijaya/ Dr Moh Hoesin General Hospital, Palembang, Indonesia

\section{A R T I C L E I N F O Keywords: \\ Coronary slow flow phenomenon \\ Fibroblast growth factor-23 \\ Endothelial dysfunction \\ Renin angiotensinogen aldosterone system \\ *Corresponding author: \\ Welly Oktaviandani \\ E-mail address: \\ oktaviandaniwelly@yahoo.co.id}

All authors have reviewed and approved the final version of the manuscript.

https://doi.org/10.32539/bsm.v5i4.379
A B S T R A C T

The phenomenon of angina chest pain without significant epicardial coronary artery stenosis, but accompanied by a slowdown in coronary blood flow is often found in patients with symptoms of acute coronary syndrome who undergoing invasive coronary angiography. This phenomenon of slow coronary blood flow is then called the coronary slow flow phenomenon (CSFP). The pathogenesis mechanism of CSFP remains unclear. The pathogenesis of CSFP is thought to be multifactorial. Endothelial dysfunction, small vessel disease, inflammation, renin system angioten sin aldosterone, atherosclerosis are thought to be involved in the pathogenesis of CSFP. Cardiovascular disease incidence and death were associated with elevated levels of Fibroblast growth factor-23 (FGF-23). High levels of FGF23 can lead to formation of blood vessel calcification, left ventricular hypertrophy, arterial stiffness, endothelial dysfunction, increased inflammatory markers and elevated levels of angiotensin II. It is suspected that FGF-23 has a role in this event other than as a regulator of bone and mineral metabolism. This literature review aims to determine the relation ship between fibroblast growth factor-23 and the pathophysiology of CSFP. Based on the broad role of FGF-23, it is possible that FGF-23 is involved in the pathogenesis of CSFP.

\section{Introduction}

Coronary Slow Flow Phenomenon (CSFP) is the result of an angiographic examination in the form of a slowdown in the filling of contrast agent injected into the coronary arteries during coronary angiography. 1,2 The incidence of CSFP is reported to be $1-7 \%$ in patients undergoing diagnostic angiography because of clinical suspicion of cardiovascular disease.2,3 This phenomenon is most common in men, smokers and patients treated with acute coronary syndromes. Slow coronary flow is associated with life-threatening arrhythmias and sudden cardiac death, possibly due to increased QTC dispersion in patients. The exact pathogenesis of CSFP remains unclear and is thought to be multifactorial. Functional and structural abnormalities of the microvasculature, endothelial dysfunction, inflammation, atherosclerosis and anatomic factors of the epicardial arteries are all involved.2,3,4 
Fibroblast Growth Factor-23 (FGF-23) is a circulating peptide hormone produced by osteocytes, osteoblasts, endocrine organs, heart, hypothalamus and thalamus. Functions to control phosphate and vitamin D metabolism. ${ }^{5}$ Several studies have reported that FGF-23 levels are associated with cardiovascular disease incidence and mortality. 6 High FGF-23 levels are associated with increased cardiovascular disease caused by vascular calcification, left ventricular hypertrophy, stiffness arterial dysfunction, endothelial dysfunction and increased inflammatory markers. 5,6 Based on these results, FGF-23 is thought to have a role in the pathogenesis of CSFP. This literature review aims to determine the relationship of fibroblast growth factor-23 to the pathophysiology of CSFP.

\section{Coronary slow flow phenomenon definition}

Coronary Slow Flow Phenomenon (CSFP) is the result of angiographic examination which is characterized by slowing of filling of distal vessels by contrast agent in the absence of significant epicardial and myocardial coronary artery stenosis. 1,2 Although various formal definitions have been proposed, CSFP essentially consists of a delay in the filling of the contrast agent injected into the coronary arteries during coronary angiography. ${ }^{2}$ Based on cross sectional study In a 2016, Ali Ghanie et al proposed that CSFP was established based on a slowdown in the emptying of the contrast agent in the coronary arteries during angiography. ${ }^{4}$ This condition that can affect one or all of the coronary arteries was first described by Tambe et al in 1972. Since then it has been known as a clinical manifestation called coronary slow flow phenomenon (CSFP), coronary slow flow syndrome, syndrome $\mathrm{Y}$ or coronary slow flow primary. Coronary Slow Flow Phenomenon has clinical implications because it is associated with clinical manifestations of myocardial ischemia, life-threatening arrhythmias, sudden cardiac death, and recurrent acute coronary syndromes. ${ }^{2}$

\section{Diagnosis and clinical manifestations clinical features}

Clinical symptoms of CSFP in more than $80 \%$ of patients experience recurrent chest pain, mostly at rest. Changes in the ECG and elevations in troponin may occur.7 Coronary Slow Flow Phenomenon is associated with life-threatening arrhythmias and sudden cardiac death. Cases of abnormal QT dispersion and ventricular arrhythmias have been reported. Clinically, this phenomenon is most common in men and young smokers and patients treated with acute coronary syndromes. ${ }^{2,3}$

Coronary Slow Flow Phenomenon often occurs in metabolic syndrome where there is an increase in total cholesterol levels, LDL cholesterol levels, fasting glucose levels and body mass index. Insulin resistance and impaired glucose tolerance are associated with the incidence of CSFP. This suggests that the underlying pathophysiological mechanisms of the metabolic syndrome and CSFP in general may be due to endothelial dysfunction. 2,3

The diagnosis of CSFP can be made semiquantitatively and quantitatively. Semi quantitative assessment using thrombolysis in myocardial infarction (TIMI) flow grade that reflects the speed and completeness of the passage of contrast injected into the coronary branch. ${ }^{7.8}$ There is a delay in filling if the distal vessel opacification takes $\geq 3$ pulses (TIMI flow grade 2). While quantitative assessment can be done using the Gibson method. There is a slowing of coronary flow according to Gibson if to achieve distal opacification, a corrected total frame count of $>27$ frames is required, with a shooting speed of 30 frames/second and a correction factor of 1.7 for Left Anterior Decending (LAD) vessels. ${ }^{8}$ 


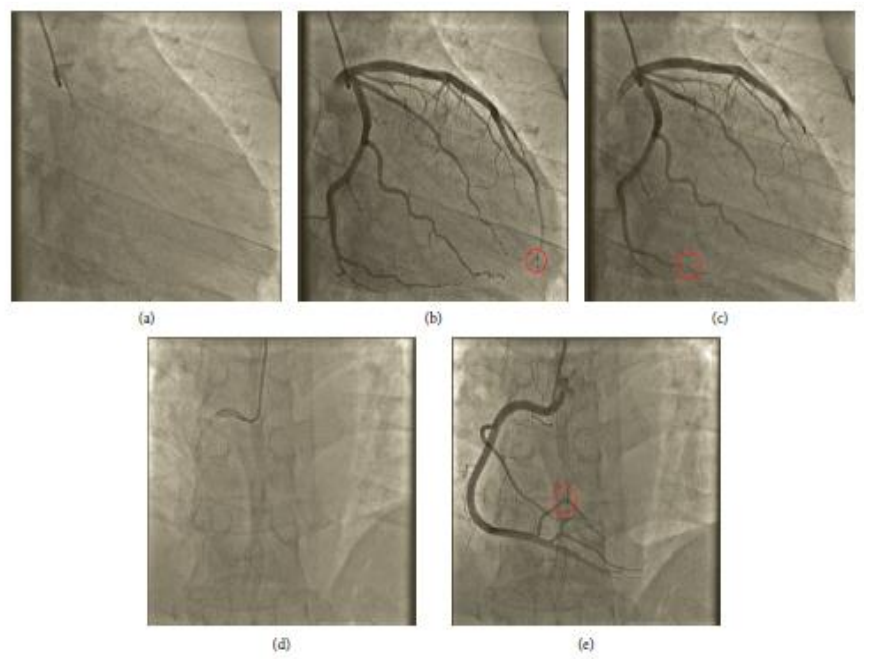

Figure 1. TIMI frame count 9

\section{Pathogenesis}

Functional and structural abnormalities of the microvasculature, endothelial dysfunction, inflammation, atherosclerosis and anatomic factors of the epicardial arteries can all be involved. ${ }^{2.3}$

\section{Microvascular disorders}

The coronary circulation consists of two compartments. The first compartment consists of epicardial vessels which are referred to as conductance vessels because they do not pose any resistance to blood flow. Compartment second consists of microvascular with size $<400 \mu \mathrm{m}$ which serves $\mathrm{m}$ engatur myocardial blood flow. Microvascular disorders are commonly involved in the pathogenesis of CSFP from the first diagnosis. ${ }^{2}$ According to some studies occur hyperplasia fibromuscular, h ipertrofi tunica media of the coronary arteries, the proliferation mi ointimal, as well as endothelial edema, thickening and degeneration pa da coronary micro vessels on CSFP. 1,2 ,7 Research Mangieri et al found a thickening of the vessel wall by shrinking the size of the vessel lumen, abnormalities of mitochondria and reduced glycogen levels in biopsy endomiok ardial left ventricle. 2,7 Beltrame et al revealed that the CSFP associated with increased tone of microvascular coronary chronically elevated characterized by oxygen saturation lower the coronary sinus and the reduced response to stimulation of endothelial se Perti cold test or the administration of acetylcholine.1,2 K ombinasi structural abnormalities that occur simultaneously on the microcirculation of the coronary thought to be the cause of the CSFP. 2

\section{Endothelial dysfunction}

Vascular endothelium plays an important role in the regulation of vascular tone, platelet activity, leukocyte adhesion, vascular smooth muscle proliferation and plays a role in the development of atherosclerosis. 2,3,9 In patients with CSFP found low levels of Flow Mediated Dilatation (FMD) from the arteries. brachial. Flow Mediated Dilatation is an endothelial-dependent process that plays a role in endothelial relaxation against increased pressure by releasing vasodilator mediators.2,10 This suggests that endothelial dysfunction is involved in the cause of CSFP. In addition, in CSFP plasma endothelin-1 levels were higher and nitric oxide was lower in nitrate. 2,10,11,12

Elevated plasma homocysteine levels and asymmetric dimethylarginine (ADMA) which is a nitric oxide synthase inhibitor were found in CSFP. Both have a detrimental effect on endothelial function. Decreased levels of adiponectin and paraoxonase activity which are significant signs of endothelial dysfunction also play a role in the pathogenesis of CSFP. 2,7,11,12

\section{Subclinical atherosclerosis}

Research Cin et al pointed - right that the CSFP 
occur thickening of the intima diffuse, extensive calcification uninteresting njang coronary vessel wall and non-obstructive atheromatous changes in coronary by using intravascular ultrasound examination technique (IVUS). ${ }^{13} \mathrm{P}$ enelitian Pekdemir et al showed sump wa most patients CSFP Memi Liki calcification throughout the epicardial artery massive koro ner. Based on these studies, CSFP may reflect non-obstructive atherosclerotic disease of the epicardial vessels along with microvascular disease. 14 Another study showed that at erosklerosis diffuse frequently found in normal coronary arteries seca ra angiography. 13.14

\section{Inflammation}

Inflammation is a contributing factor to several cardiovascular conditions and is found in CSFP. Research by Li et al showed that in CSFP plasma levels of high-sensitivity C-reactive protein (hsCRP) and interleukin-6 were increased. In addition, CSFP is associated with increased levels of plasma soluble adhesion molecules, such as intercellular adhesion molecule-1(ICAM-1), vascular cell adhesion molecule-1 (VCAM-1) and E-selectin.15 Other inflammatory markers such as uric acid levels have also been shown associated with the incidence of CSFP. Overall abnormalities in inflammatory parameters can be indicators of endothelial dysfunction and contribute to CSFP. 7,15

\section{Anatomical factors}

The pattern of blood flow in the epicardial coronary arteries depends on the shape, size and movement of the blood vessels. Impaired blood flow occurs in the arteries with shapes and sizes such as indentations, branches and bifurcations. In these areas a slower rate of blood flow tends to occur. ${ }^{16,17}$ Using CT coronary angiography it can be seen that in patients with CSFP, the angulation of the main coronary arteries of the aorta is seen to be smaller. 18

Katarci et al. conducted a case-control study to examine the correlation between the anatomic properties of the coronary arteries and the incidence of CSFP. The results showed that CSFP was associated with higher tortuosity and more distal branches of the coronary arteries. With the results of these studies it can be concluded that certain anatomic properties of the coronary arteries can predispose to disruption of coronary flow and endothelial damage which can ultimately lead to CSFP. 18

\section{Interaction of local and systemic conditions}

The combination of morphological and functional abnormalities in the small vessels and epicardial coronary arteries is currently thought to be the etiologic cause of CSFP. In addition, another characteristic of CSFP is that it is often associated with more extensive vascular abnormalities. Karakaya et al's study found cerebral blood flow at a lower rate in patients with CSFP. Endothelial abnormalities are a common process that affects coronary and peripheral blood vessels. 19

Camsari et al using intravascular ultrasound found that there was a significant relationship between coronary intima-media thickness (BMI) and carotid BMI.20 In contrast, aortic distensibility and stretch, which are other predictors of subclinical atherosclerosis, were lower in patients with CSFP. 21 Based on studies it can be concluded that early atherosclerosis is not limited to the coronary circulation but also extends to the large peripheral arteries in patients with CSFP. Simultaneously, CSFP may be caused by interactions between local coronary artery conditions and systemic pathophysiological factors. 22

\section{Renin angiotensin aldosterone system and cardiovascular}

The renin angiotensin aldosterone system (RAAS) is a regulator of blood pressure and a determinant of target organ damage. This system is directly involved in the regulation of blood pressure, fluid volume and vascular response to injury and inflammation.23 Improper activation of the RAAS causes hypertension, fluid retention, inflammation, thrombotic and atherogenic which can contribute to long-term target organ damage.24 This system controls fluid and electrolyte balance through coordinated effects on the heart, blood vessels and kidneys.23 When 
overexpression of the RAAS occurs, the RAAS plays a major role in cardiovascular disease through increased blood volume, increased arterial pressure, fibrosis, prothrombotic conditions and the formation of vascular lesions. Overexpression of the RAAS causes a variety of deleterious vascular effects. 24

The effect of the RAAS on target tissues is largely influenced by circulating and tissue angiotensin II (AII), although aldosterone, renin, and some breakdown products of angiotensin I are also involved.23,24 Angiotensin II is a major effector of the RAAS because of its vasoconstrictor effect. Angiotensin II can play a direct role in cardiovascular damage through the processes of cell growth, inflammation and fibrosis. Angiotensin II causes endothelial dysfunction by activating important transcription factors, especially nuclear factor-k $\beta$ (NFk $\beta)$, thereby inducing proinflammation in vascular smooth muscle. These conditions all cause vascular remodeling. 24,25 Based on the broad role of the RAAS on the cardiovascular system, it is currently suspected that the RAAS has a role in the pathophysiology of CSFP. 4

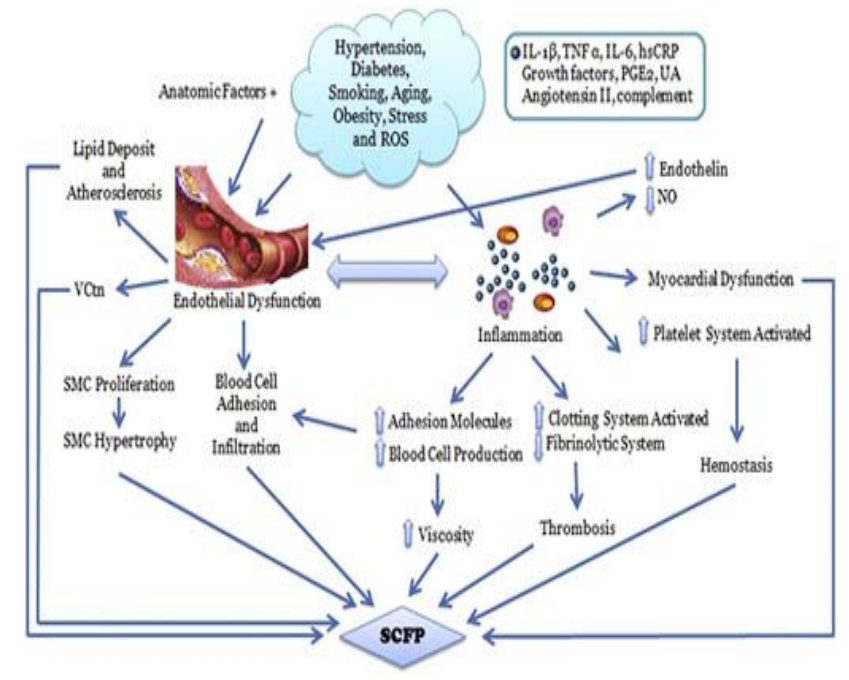

Figure 2. Pathophysiology of coronary slow flow phenomenon 26

\section{Fibroblast growth factor}

Fibroblast growth factor consists of 22 family members. The FGF family is phylogenetically divided into seven different subfamilies.27 Based on its mechanism of action, FGF is divided into paracrine/canon, endocrine and intracrine. The FGF family included in the paracrine is FGF-1 to 10 , FGF16 to 18, FGF-20 and FGF-22. The paracrine group works by binding and activating the FGF receptor tyrosine kinase (FGFR) with heparin sulfate as a cofactor and canonical. Meanwhile, the endocrine group consists of FGF 15/19, 21 and 23 which work systemically through hormonal pathways that are dependent or not dependent on the FGF receptor (FGFR). All endocrine FGFs require and Klotho coreceptors for high affinity binding to FGF receptors expressed in target cells. Fibroblast growth factor binds to the FGF receptor via heparin/heparan sulfate with very low affinity. The reduced binding affinity of heparin allows endocrine FGFs to perform their functions at distant sites. The intracrine FGF group is not secreted and acts on the same cell via an FGF receptor independent pathway. Included in the intracrine group are FGF-11 to FGF-14.28 Fibroblast growth factors are not all expressed endogenously in the human heart. Some of the FGFs that act on the heart include FGF-2, FGF-3, FGF-8, FGF-9, FGF-10, FGF-16, FGF-15/19, FGF-21 and FGF-23 which act in a paracrine or endocrine manner. to stimulate physiological or pathological pathways in the development of heart health and disease. 29

In mammals there are seven FGFRs produced from four different FGFR genes (FGFR1, FGFR2, FGFR3, FGFR4) with different FGF-binding affinities. Paracrine growth factor fibroblasts bind to heparan sulfate to 
form the FGF/FGFR/heparan sulfate complex. 28 Endocrine growth factor fibroblasts bind to either klotho or -klotho to form the FGF/FGFR/klotho complex, both of which directly phosphorylate the intracellular tyrosine kinase domain of FGFR. After FGFR activation, the substrate FGFR-2a (FRS2a) is activated thereby stimulating the activation of the renin angiotensin aldosterone system (RAAS), rat sarcoma protein (RAS), mitogen-activated protein kinase (MAPK), phosphoinositide 3-kinase (PI3K), serine/threonine kinase (AKT) and signal transducer and activator of transcription (STAT) in each cell. In addition, phospholipase $\mathrm{C} \gamma$ (PLCY) can also be phosphorylated due to FGFR activation, thereby causing calcium-dependent activation of cell signaling. In the cardiovascular system, FGFR-1c, FGFR-2b, FGFR-2c, FGFR-3c and -klotho were physiologically expressed at very high levels whereas FGFR-1b, FGFR3b, FGFR-4 and -klotho were only expressed with lower rate. 30

\section{Fibroblast growth factor-23}

Fibroblast growth factor-23 (FGF-23) is a phospaturic hormone produced and expressed mainly by osteocytes to maintain phosphate and mineral homeostasis. Fibroblast Growth Factor-23 is a relatively large protein $(32 \mathrm{kDa})$ compared to other FGF members. ${ }^{31,32}$ The FGF-23 gene is located on the human chromosome at 12 p3.3 and consists of three separate exons and two introns. Mostly expressed by osteocytes and osteoblasts in bone and is also present in pericyte-like cells around the sinus venosus bone marrow, ventrolateral thalamic nucleus, thymus and lymph nodes. 33 Fibroblast Growth Factor-23 is biologically active in full and can be cleaved into $\mathrm{N}$ - and C-terminal fragments. The FGFR site is located at the $\mathrm{N}$-terminal end whereas the -klotho site is located at the $\mathrm{C}$-terminal. The $\mathrm{N}$-terminal fragment contains a signal peptide for secretion indicating that FGF-23 is a member of the FGF family that circulates in the blood endocrine effects rather than autocrine or paracrine effects. $31,32,34$

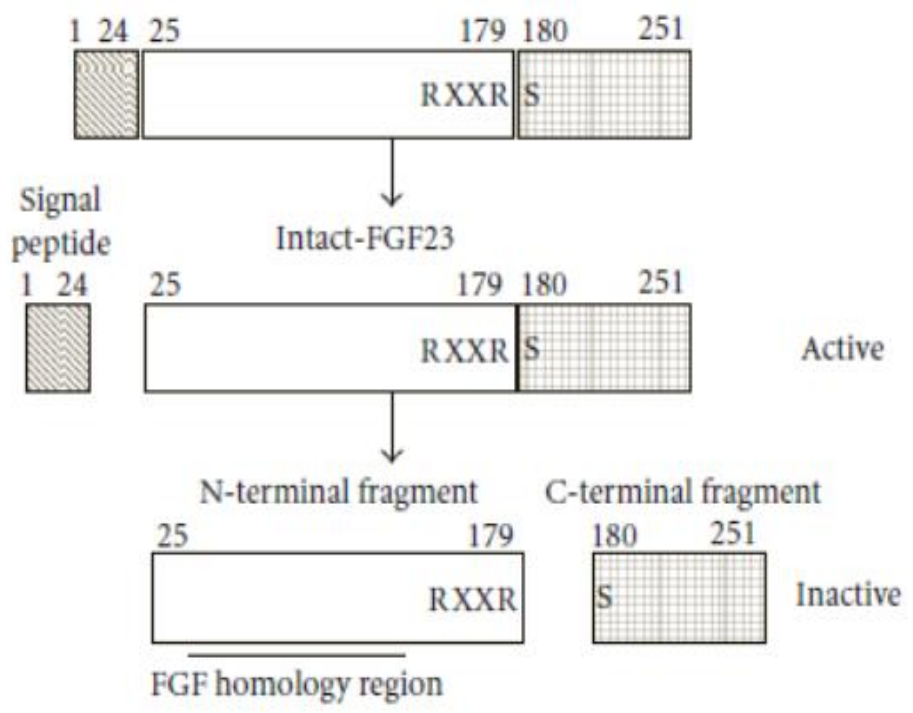

Figure 3. FGF-23 protein structure ${ }^{31}$
FGF-23 expression is almost absent in the central nervous system, non-reproductive endocrine system and metabolic system and is only minimally expressed in the digestive system, immune system, reproductive system, and cardiovascular system in healthy adults. Under pathological conditions, FGF23 is known to be excessively elevated in bones, heart, liver and kidneys. $35,36,37$

\section{Several factors play a role in the regulation of fibroblast growth factor-23}

Since the discovery of FGF-23 many studies have been carried out aimed at evaluating the factors that regulate the production and cleavage of FGF-23. 


\section{a. Vitamin D}

Administration of vitamin D increased FGF-23 in humans and mice. Calcitriol increases intestinal absorption of phosphate and calcium, both of which can also increase FGF-23 production. Vitamin D acting on its specific receptor (VDR) stimulates the promoter of the FGF-23 gene and is an effect that is independent of serum phosphate and calcium levels. In addition, vitamin D produced locally in bone cells tends to regulate FGF-23 production. 33

b. Phosphate

Phosphate receptors have not yet been discovered but it is proven that the presence of phosphate, even in the absence of high serum phosphate levels can stimulate FGF-23 production. In CKD patients and patients on dialysis, high serum phosphate concentrations are associated with elevated levels of FGF23 which are then associated with increased C-reactive protein (CRP). Phosphate levels contribute $70 \%$ to high levels of FGF 23.33

c. Calcium and PTH

Parathyroid hormone (PTH) stimulates the transcription of FGF23 by influencing calcitriol. Parathyroid hormone activates nuclear receptor nurr1, which is the initial mechanism by which PTH increases FGF-23. In dialysis patients, FGF-23 is positively correlated with serum phosphate and PTH levels and inversely with serum calcium concentrations. Hypocalcemic conditions are thought to reduce FGF-23 levels in order to prevent a decrease in calcitriol which can worsen hypocalcemic conditions. In healthy people and CKD patients, consumption of a calcium-fortified diet was associated with an increase in FGF-23 33

d. Inflammation and Iron Deficiency

Inflammation and FGF-23 production have a two-way relationship because inflammation and increased FGF23 are associated with death. Acute or chronic inflammation can lead to transcription and cleavage of FGF-23. Induction of acute inflammation in mice can increase bone FGF-23 mRNA expression. Likewise, the induction of chronic inflammation is followed by an increase in FGF-23.33

e. Aldosterone

Research in recent years has demonstrated an association between FGF-23 and aldosterone regulation. By activating the FGFR1/a-Klotho/ERK pathway, FGF-23 increases sodium reabsorption via sodium/chloride co-transporters in the distal tubule, resulting in increased fluid volume and increased blood pressure. Simultaneously, aldosterone increases FGF23 secretion and further FGF-23 causes an increase in angiotensin II expression in cardiac myocytes. ${ }^{33}$

\section{Functions of fibroblast growth factor-23}

The specific function of FGF-23 depends on the presence of the FGF receptor (FGFR). Under physiological conditions, FGF receptor-1c (FGFR1c) is the most important FGFR for FGF-23.38 signaling. 38 The principle of intracellular FGF-23 signaling is dimerization of FGF-23 receptors and activation of intrinsic FGFR tyrosine kinase activity by transphosphorylation. The main substrates for FGFR kinase are substrate $2 a$ (which stimulates the RAAS, MAPK, P13-AKT pathway) and phospholipase Cג1 (which stimulates protein kinase $\mathrm{C}$ and activation of calcineurin-nuclear factor activated T cells (NFAT)). 39 The main target organ of FGF-23 is where FGF-23 acts on the renal tubules to reduce phosphate reabsorption. In the kidneys, FGF-23 acts via the FGFR1c/aklotho/MAPK signal.40,41 Its main function in mineral metabolism is to reduce phosphate reabsorption by suppressing the sodium-phosphate transporters NaPiIIa and NaPi-IIc.5,25 Functions of FGF-23 the other is to suppress the synthesis of active vitamin $D$ by suppressing the synthesis of 1a-hydroxylase and upregulation of 24-hydroxylase in the kidney, causing low serum levels of $1,25(\mathrm{OH})_{2} \mathrm{D} 3$. In the parathyroid glands, FGF-23 works by inhibiting the secretion of parathyroid hormone. Other target organs are the liver, heart, bones and the immune system. 5,25,42 


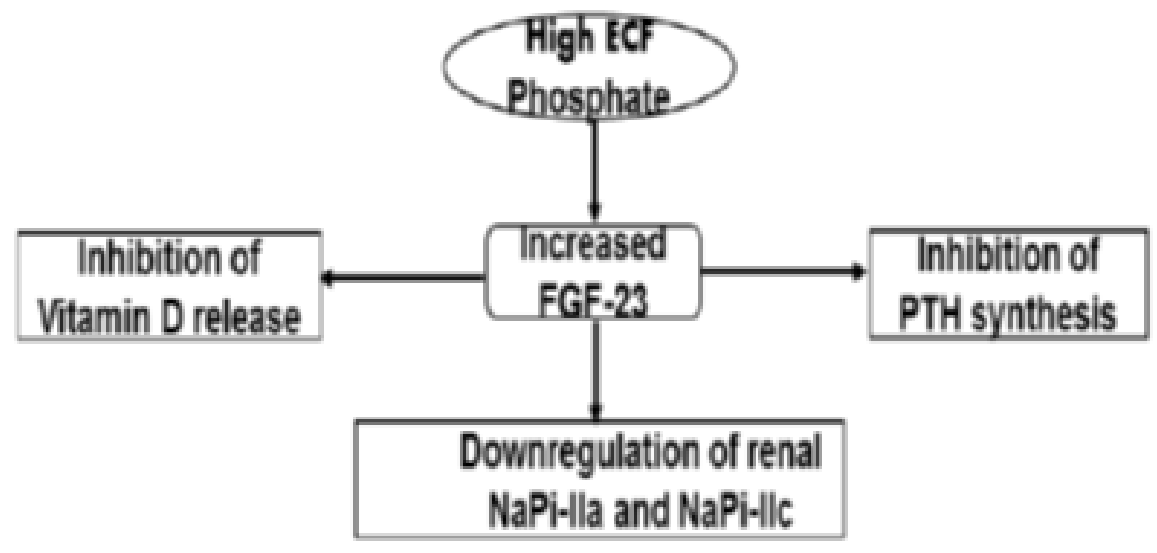

Figure 4. Mechanism of action of fibroblast growth factor-23 5

\section{Physiological mechanism of fibroblast growth factor-23}

Fibroblast growth factor-23 is a hormone produced mainly by osteocytes that functions to suppress phosphate reabsorption and vitamin $D$ synthesis in the kidneys. At normal levels the endocrine action of FGF23 in the kidney depends on -klotho as a co-receptor because the high affinity binding of FGF-23 to the FGFR-1c receptor requires the presence of $\alpha$-klotho coreceptors on target cells. Most -clotho is expressed in the kidney and choroid plexus, parathyroid gland, pituitary gland, placenta, skeletal muscle, pancreas and testes. Co-factor a-klotho is actually required to recognize FGF-23 signals but the discovery of an independent pathway for FGF-23 transduction does not necessarily require -klotho as a co-factor. For example, in cardiomyocytes there is no expression of $\alpha$ klotho so that FGF-23 in cardiomyocytes does not require -klotho. $39,42,43$

Excessively elevated circulating levels of FGF-23 can cause phosphaturia in individuals with normal renal function. Research in recent years has demonstrated that in addition to the physiological role of FGF-23 in the control of 1a-hydroxylase expression, FGF-23 is also an important stimulator of calcium and sodium reabsorption in the distal renal tubule and as a paracrine autoregulator of alkaline phosphatase expression and mineralization in bone. ${ }^{44}$

Physiological function of fibroblast growth factor23 in the kidney
Studies in mice have revealed that the most important physiological function of FGF-23 is not its phosphaturic function but its repressor role in the transcriptional control of renal 1a-hydroxylase which is a major enzyme for $1,25(\mathrm{OH})_{2} \mathrm{D} 3$ synthesis. Expression of 1a-hydroxylase is mainly in the proximal renal tubule. In the absence of FGF-23 or -klotho ligands, it can interfere with the control of endocrine function of 1a-hydroxylase transcription, resulting in increased expression and inappropriate enzyme activity. The result of overexpression of 1a-hydroxylase is increased levels of $1,25(\mathrm{OH})_{2} \mathrm{D} 3$ which causes hypercalcemia, hyperphosphatemia, ectopic calcification, impaired bone mineralization, and premature death in mice with low levels of FGF-23 and -klotho. 42

The main function of $1,25(\mathrm{OH}) 2 \mathrm{D} 3$ in mineral metabolism is to stimulate the absorption of calcium and phosphorus in the intestine. An important role of $1,25(\mathrm{OH})_{2} \mathrm{D} 3$ in relation to $\mathrm{FGF}-23$ and -klotho is ablation of vitamin D signaling so as to maintain FGF 23 and -klotho. In individuals with increased vitamin D levels, there will be a decrease in the function of FGF 23.42 Expression of 1a-hydroxylase is mainly found in the proximal renal tubule. The proximal and distal tubules of the kidney express -klotho as well as FGFR1, FGRF-3, FGFR-4 and only a small amount of FGFR2. All FGFRs are receptor tyrosine kinases that initiate the intracellular phosphorylation cascade after ligandinduced binding. In rats with deletion of FGFR-1 in the proximal renal tubules, there is no suppression of the production of $1,25(\mathrm{OH})_{2} \mathrm{D} 3$ by FGF-23. Therefore, 
FGFR-1 may be the dominant FGFR in the renal tubules that play a role in suppressing the synthesis of 1-hydroxylase 1,25. $(\mathrm{OH})_{2} \mathrm{D} 3 . \quad$ FGF-23-mediated suppression of 1a-hydroxylase transcription involves activation of extracellular signal kinase 1 and 2 upregulated extracellular signal-regulated kinase 1/2 (ERK1/2) but the precise signaling pathway in ERK1/2 is unknown., $40,41,42$

The role of FGF-23 in inhibiting phosphate reabsorption in the proximal renal tubule occurs via a signaling cascade involving the -klotho/FGFR1c receptor complex, ERK1/2, and serum/glucocorticoidregulated kinase-1 (SGK-1) where FGF-23 signaling induces phosphorylation of protein structure $\mathrm{Na}+/ \mathrm{H}+$ exchange regulatory cofactor-1 (NHERF-1) which causes internalization and degradation of sodium phosphate cotransporters NaPi-IIa and NaPi-IIc.5,25,42

In addition to its role in vitamin $\mathrm{D}$ and phosphate metabolism, FGF-23 also directly affects calcium and sodium metabolism in the distal renal tubule. In renal distal tubular epithelium FGF-23 regulates apical membranes rich in transient receptor potential vanilloid-5 (TRPV5) calcium channels and sodium chloride cotransporter NCC via a signaling cascade involving ERK1/2, SGK1 and with-no-lysin kinase-4. In mice with FGF-23 deficiency and klotho, it is characterized by downregulation of distal tubular TRPV 5 and membrane expression of NCC which causes renal excretion of calcium and sodium and as a feedback an increase in PTH and aldosterone levels. These findings suggest that maintaining calcium and sodium balance is part of the physiological function of FGF-23 in the distal renal tubule. ${ }^{42,45}$

It is currently known that $\mathrm{PTH}$ and $\mathrm{FGF}-23$ regulate the expression of $1 \mathrm{a}$ and 24-hydroxylase (CYP24A1) reciprocally. Fibroblast growth factor-23 suppresses 1a-hydroxylase, but stimulates 24-hydroxylase expression. Parathyroid hormone has the opposite effect. The functions of parathyroid hormone and FGF23 in the proximal and distal tubules of the kidney partially overlap. Both hormones inhibit phosphate reabsorption in the proximal renal tubule by targeting NHERF-1 phosphorylation and increase calcium reabsorption in the distal renal tubule by targeting
TRPV-5 expression. The target of PTH and FGF-23 molecules in the kidney is the same although the signaling mechanism is different. One study revealed that the absence of FGF-23 signaling in mice with low levels of FGF-23 caused partial resistance of the kidneys to phosphaturia. Likewise, the reduced PTH signaling state in individuals with hypoparathyroidism has been shown to stimulate partial resistance to phosphaturia by FGF-23. It is therefore one of the important physiological functions of FGF-23, possibly as a normal response to PTH signaling in the kidney as well as in bone. ${ }^{42,45}$

\section{Physiological function of fibroblast growth factor- 23 in bone}

Fibroblast growth factor-23 has physiological functions in bone in the process of bone mineralization and in hematopoiesis. Murali et al reported that FGF 23 is a potent inhibitor of non-specific alkaline phosphatase (TNAP) mRNA transcription in bone cells independent of the klotho co receptor. Non-specific tissue alkaline phosphatase is very important in the regulation of bone mineralization by inhibiting mineralization pyrophosphate inhibitors produced by osteoblasts to prevent premature mineralization of osteoid. 46

Shalhoub et al reported that FGF-23 suppressed TNAP expression in mouse osteoblast cells by binding to FGFR1 and this effect was enhanced in the presence of klotho. Klotho expression in bone is very low, therefore klotho expression in bone is not sufficient to increase FGF-23 binding to FGFR in osteoblasts and osteocytes. So it is estimated that due to the production of FGF-23 in osteocytes, the concentration of FGF-23 in the canalicular system is high enough to function as an auto/paracrine. Therefore, FGF-23 produced in bone not only contributes to impaired mineralization but may also function as a physiological inhibitor of bone mineralization by downregulating TNAP expression. 47

\section{Physiological function of fibroblast growth factor- 23 in other organs}

The description of FGF-23 was first actually found 
in the thalamic nucleus in the brain but data on the possible function of FGF-23 in the brain are sparse. It has been reported that high concentrations of FGF-23 can disrupt neuronal branching and increase synaptic density in cultured hippocampal neurons but very little is known about its potential physiological function. 42

The parathyroid gland is one of the organs that has a lot of -clotho expression, making it a potential target organ for FGF-23. However, mice with parathyroid clotho deletion showed specific normal PTH levels. Although high concentrations of FGF-23 can suppress PTH secretion, FGF-23 does not have an important role in the physiological regulation of PTH secretion. ${ }^{31}$

The heart is the target tissue for elevated levels of FGF23. In CKD patients, ventricular hypertrophy may occur due to elevated FGF23 levels. Fibroblast growth factor-23 was not expressed in normal liver and normal heart function in FGF-23/VDR mutant mice. These findings suggest that FGF-23 has no functional role in the heart under physiological conditions. Overall, there is little evidence that FGF-23 has a role

in other organs under normal conditions other than the kidneys and bones. 25

\section{Role of fibroblast growth factor-23 on coronary slow flow phenomenon}

Several studies have shown an association between FGF-23 levels and heart disease. Coronary heart disease has a very strong relationship with FGF-23 levels. High levels of FGF-23 are known to be associated with vascular dysfunction, atherosclerosis and cardiovascular disease in individuals with normal renal function. 6 In most prospective cohort studies each level of $20 \mathrm{pg} / \mathrm{ml} \mathrm{FGF-23} \mathrm{is} \mathrm{associated} \mathrm{with} \mathrm{a} \mathrm{risk} \mathrm{of}$ coronary artery disease (CAD) of approximately $14 \%$. In addition, there was a significant relationship between serum FGF-23 and the presence of CAD as well as some vascular stenosis associated with FGF-23. The most common cause of heart failure is CAD and is associated with FGF23 levels. ${ }^{48}$

In CKD patients with high FGF-23 levels, the incidence of heart failure is 2 times higher than those with low FGF-23 levels. This increase in incidence remains significant in patients without $\mathrm{CKD}$ although less strongly. This is indicated by the results of largescale cohort studies that the incidence of heart failure is 1.75 times higher in individuals with high FGF-23 levels and remains significant in individuals without CKD. 49

Increased levels of FGF-23 cause an increase in intracellular and extracellular calcium. Increased intracellular calcium levels in the left ventricle of the heart activate the calcineurin-nuclear factor activated $\mathrm{T}$ cells (NFAT) signaling pathway that causes left ventricular hypertrophy. 5 Meanwhile, increased extracellular calcium levels due to secondary hyperparathyroidism can cause vascular calcification. High levels of FGF-23 directly activate the expression of $\mathrm{NaCl}$-transporter (NCC) in the distal renal tubule, triggering the RAAS system and initiating oxidative stress. 33

\section{The role of fibroblast growth factor-23 and left ventricular hypertrophy}

Fibroblast growth factor-23 is thought to have a role in the occurrence of left ventricular hypertrophy (LVH). Faul et al showed that the pathological effect of FGF-23 on cardiac hypertrophy was mediated by FGFR activation of the calcineurin-nuclear factor activated $\mathrm{T}$ cells (NFAT) signaling pathway. Calcineurin is a protein that is activated by calcium-calmodulin bonds. Calcineurin is a serine/threonine-specific phosphatase that is activated by persistently elevated intracellular calcium concentrations. As a phosphatase, the activation of calcineurin dephosphorylate which is a member of NFAT is expressed in the myocardium thereby activating the hypertrophic pathway. 49

Toucberry et al that FGF-23 directly regulates intracellular calcium concentration in the myocardium. This condition may have a role in activating the calcineurin-NFAT pathway and thus a possible role for FGF-23 in the pathogenesis of LVH. 50

\section{Fibroblast growth factor-23 and endothelial dysfunction}

Endothelial dysfunction is a systemic pathological condition that can be defined as the result of an imbalance between vasorelaxation and vasoconstrictor 
actions. This imbalance is mainly due to reduced NO levels and/or increased reactive oxygen species (ROS). ${ }^{51}$ Endothelial dysfunction is considered to be one of the early mechanisms involved in the atherosclerosis process. In CKD, endothelial dysfunction is defined as elevated von Willebrand factor, low NO levels, elevated inflammatory cytokines and C-reactive protein (CRP). Reduced levels of NO cause a decrease in the ability of blood vessels to vasodilate and can increase the risk of hypertension. Schiffrin et al revealed that the interaction of endothelial dysfunction, inflammation and dyslipidemia can increase the acceleration of atherogenesis. The endothelium produces NO which is an anti-atherogenic molecule. Decreased levels of NO are the result of increased levels of asymmetric dimethylarginine (ADMA). Asymmetric dimethylarginine (ADMA) is released through protein hydrolysis. ADMA is eliminated by renal excretion but its elimination is mainly due to metabolic degradation by dimethylarginine dimethylaminohydrolase (DDAH). 52

DDAH inhibition is regulated by ROS and oxidative stress in endothelial cells, thereby increasing ADMA levels. ROS production in endothelial cells can be stimulated by FGF-23. The role of ADMA is not only as an inhibitor of NO synthesis but also as an inhibitor of the entry of L-arginine into cells to reduce NO synthesis. ADMA levels increase with worsening renal function and the highest levels are found in dialysis patients. Increased ADMA indirectly causes vasoconstriction, inhibits vasodilation of blood vessels, causing platelet adhesion and aggregation. This condition can lead to the release of superoxide radicals, formation of peroxynitrite and nitration of tyrosine which causes vascular endothelial damage. 52

Fibroblast growth factor-23 has a role in vascular function. Excessive levels of FGF-23 are associated with impaired vascular vasodilation. The study of Verkaik $\mathrm{M}$ et al, found that increased levels of FGF-23 cause endothelial dysfunction through peripheral arterial resistance. Fibroblast growth factor-23 injected in experimental mice caused a reduction in the vascular vasodilation response. Then the administration of FGF-23 antibody in experimental mice could restore the vasodilation response. 53 In addition, administration of FGF-23 in experimental mice could cause an increase in ADMA levels. Therefore endothelial dysfunction caused by FGF-23 occurs due to reduced levels of NO caused by increased levels of ADMA. Isabell et al revealed that the vasoconstrictor effect of FGF-23 is related to the levels of ROS in smooth muscle cells (SMC). ROS activation is thought to be the sole role of FGF-23 in endothelial dysfunction. 51

NO levels are partly dependent on superoxide. Silwal N et al's study showed that FGF23 plays a role in reducing NO levels in aortic tissue by increasing superoxide. Increased levels of FGF-23 can increase endothelial superoxide levels and by inhibiting superoxide can prevent endothelial dysfunction caused by FGF23. The study concluded that the role of FGF 23 in endothelial dysfunction was due to an increase in superoxide levels, resulting in reduced NO levels. 54

\section{The role of fibroblast growth factor-23 in the renin angiotensin aldosteron system}

In the classic RAAS pathway, renin is secreted from the juxtaglomerular apparatus of the kidney and acts on circulating angiotensinogen precursors to produce angiotensin I. Renin hydrolyzes the binding of angiotensinogen to angiotensin I. Angiotensin converting enzyme (ACE) present in the vascular endothelium and lungs, converts angiotensin $\mathrm{I}$ to angiotensin II. Angiotensin II is produced in the heart, kidneys and brain. In addition, it is also produced by a non-ACE pathway involving chymase, cathepsin G, enzymes such as kallikrein and endopeptidase and has an effect on target tissues that is even greater than the effect of AII produced through the ACE pathway. 24,25

Angiotensin II acts on the heart and kidneys by binding to type 1 (ATR1) and type 2 (ATR2) G protein receptors. Angiotensin II is a vascular vasoconstrictive peptide. Angiotensin II will then stimulate aldosterone secretion from the adrenal cortex. 24

Angiotensin II is the main effector of the RAAS. Angiotensin II has a number of rapid effects such as vasoconstriction, increased aldosterone secretion, 
increased thirst and antidiuretic hormone release, increased myocardial contractility and increased sympathetic nervous system activity. This effect is an integrated response to the presence of decreased intravascular volume. This situation if it occurs in the long term can cause changes such as vascular smooth muscle hyperplasia, myocardial hyperplasia, deposition of extracellular matrix components and cause blood vessels to be more sensitive to vasoconstriction. Change is a structural remodeling of the cardiovascular system as compensation for prolonged contraction. 24

Angiotensin II has non-hemodynamic effects that also play a role in cardiovascular disease. Nonhemodynamic effects of angiotensin II that play a role in the development of cardiovascular disease such as upregulation of cytokines, cell adhesion molecules and profibrotic growth factors such as stimulating the expression of transforming growth factor $\beta$ (TGF $\beta$ ), stimulating plasminogen activator inhibitor-1 (PAI-1), stimulating extracellular matrix protein synthesis, stimulating inhibitor of plasminogen activator-1 (PAI-1) and causes activation and infiltration of macrophages. Plasminogen activator inhibitor-1 (PAI-1) is produced by endothelial and vascular smooth muscle cells (VSMC). Plasminogen activator inhibitor-1 (PAI-1) is a major inhibitor of the fibrinolytic system and a potent anti-thrombolytic factor has been identified as a contributing factor in the pathogenesis of glomerulosclerosis and tubulointerstitial fibrosis. 24,25

Angiotensin II also stimulates oxidative stress. The expression of nicotinamide adenine dinucleotide phosphate (NADPH) produces superoxide anion which is important in signal transduction of angiotensin II against oxidative stress in the vascular, glomerulus and distal nephron of the kidney. In addition to having a direct effect on free radicals, angiotensin II can also reduce NO levels and thereby exacerbate angiotensin II-induced vasoconstriction. ${ }^{24}$

Angiotensin II acts in many tissues such as the kidneys, heart, blood vessels, brain, and lymphatic organs through binding and activation of receptors. These angiotensin receptors are divided into two distinct classes: type 1 receptors (ATR1) and type 2 receptors (ATR2). The dominant cardiovascular effect of angiotensin II is mediated by ATR1. Type 1 receptors predominate in most tissues. In contrast, ATR2 expression is highest during fetal development and decreases in most tissues to very low levels in adults. In general, the ATR2 function tends to be inversely proportional to ATR1.24,25

Angiotensin II type 1 receptors are known to play an important role in the cardiovascular system which has vasoconstrictive effects, increased blood pressure, water and salt retention, growth promotion, fibrosis and inflammation in physiological and pathological conditions. One of the well-known functions mediated by ATR 1 is to cause potent vasoconstriction. This effect is mediated by the direct effect of ATR1 in VSMC along with the indirect effect of activation of the ATR1 pathway in the peripheral central nervous system. In addition, it is known that the AT1 receptor has effects on inflammation and immune responses by modifying cytokine production and inflammatory cell migration.

Stimulation of the angiotensin II type 1 receptor by angiotensin II activates phospholipase C, which hydrolyzes phosphatidlinositol-4,5-bisphosphate to produce inositol-1,4,5-triphosphate and diacylglycerol. Inositol-1,4,5-triphosphate triggers the release of calcium from intracellular stores resulting in an increase in intracellular calcium. Angiotensin converting enzyme 2 (ACE2) is a type 1 integral membrane glycoprotein found in most tissues, with highest expression in the kidney, endothelium and heart. In the cardiovascular system ACE2 is probably more important than ACE in regulating angiotensin and angiotensin II levels. ${ }^{23}$

Angiotensin converting enzyme II is an exopeptidase that catalyzes the conversion of angiotensin I to angiotensin 1-9 and catalyzes the conversion of angiotensin II to angiotensin 1-7. Angiotensin 1-7 and 1-9 act as vasodilators and have a blood pressure lowering effect, respectively. In the heart, ACE- 2 is the main pathway in the metabolism of angiotensin II. 5

ACE-2 deficiency can cause accelerated cardiac hypertrophy and progression of cardiac fibrosis, resulting in diastolic dysfunction and pressure overload. In addition, the role of $\mathrm{ACE}-2$ is to reduce 
circulating levels of angiotensin II rather than its role in increasing angiotensin 1-7 so that inhibition of ACE2 expression will cause an increase in angiotensin II levels. 5,25

The role of FGF-23 in the RAAS by inhibiting the expression of angiotensin converting enzyme 2 (ACE2). 5 The resulting effect of suppression of ACE-2 expression by FGF-23 is an increase in intracellular calcium levels and vascular vasoconstriction. In addition, renin is suppressed by $1,25(\mathrm{OH})_{2} \mathrm{D} 3$ so that it can prevent RAAS activation. The effect of FGF-23 suppresses the metabolism of $1,25(\mathrm{OH}) 2 \mathrm{D} 3$ in the kidney. Therefore, there is an opinion that FGF-23 also stimulates the RAAS indirectly through 1,25(OH)2D3 inhibition. However, the mechanism by which FGF-23 interacts with the RAAS in the heart, causing cardiac remodeling and impaired diastolic function, is still unknown. ${ }^{24,25}$

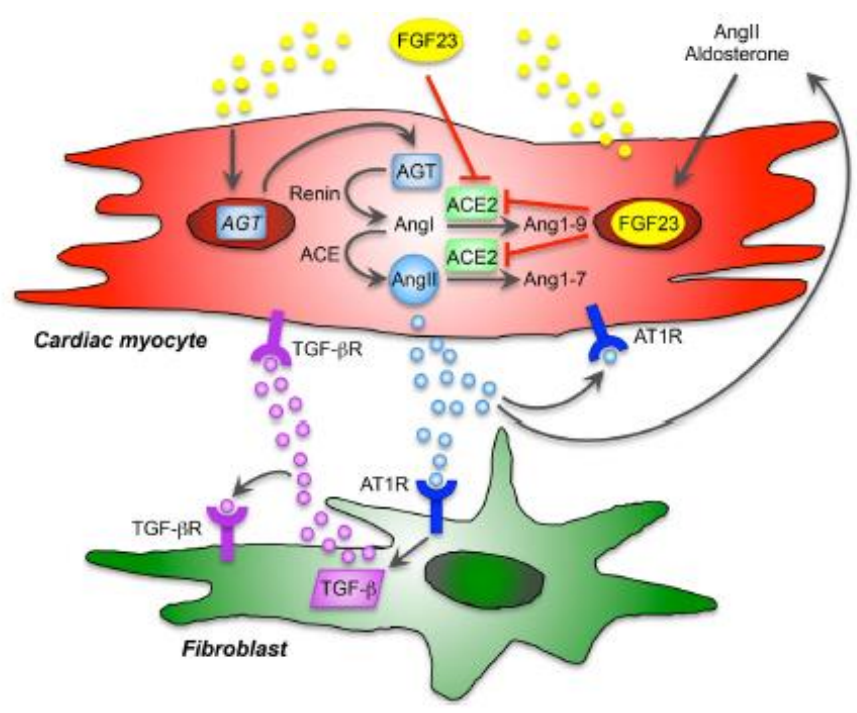

Figure 4. Effect of FGF-23 on RAAS 25

\section{Fibroblast growth factor-23 and inflammation}

Cardiac hypertrophy and myocardial fibrosis can be caused by pro-inflammatory cytokines such as TNF- $\alpha$, IL-6 and pro-fibrotic molecules including TGF- $\beta$ and angiotensin II. These proinflammatory cytokines were independently associated with FGF-23 levels in patients with CKD. Fibroblast growth factor-23 directly stimulates hepatic pro-inflammatory cytokine secretion in experimental mice. This suggests a relationship between FGF-23 and chronic inflammation. Chronic inflammation in cardiac fibroblasts is known to induce FGF-23 expression in the heart and suggests a paracrine signaling mechanism of chronic inflammation that stimulates cardiac FGF-23.25,33.41

The cytokine oncostatin M (OSM), which belongs to the IL-6 family, is involved in various biological processes such as inflammation and ischemic heart disease. The cytokine oncostatin $M$ is known to be a major mediator of cardiac remodeling. Upon binding to the receptor heterodimer comprising gp130 and the OSM- $\beta$ receptor, OSM induces the Janus Kinase/STAT, MAPK and PI3K/AKT signaling pathways. This signaling pathway is also known to be induced by the FGF/FGFR complex. Richter et al. found that FGF-23 was present in cardiac myocytes of patients with ischemic cardiomyopathy, myocarditis and dilated cardiomyopathy. Adult mouse cardiac myocytes also express FGF-23 at the mRNA level induced by OSM. Inflammation in rat heart failure caused by macrophage infiltration in the myocardium showed an increase in the expression and secretion of FGF-23 in cardiac myocytes. This condition is associated with macrophage infiltration and activation of the cardiac OSM receptor cascade leading to cardiac myocyte remodeling with increased smooth muscle actin expression, STAT3 phosphorylation, induction of atrial natriuretic peptide (ANP), and destrin. These findings 
suggest that FGF-23 is endogenously expressed in cardiac myocytes in inflammatory heart failure in humans and mice and is associated with macrophage infiltration and OSM receptor activation. ${ }^{25,41}$

Expression of FGF-23 is also present in M1 macrophages and is specifically regulated by

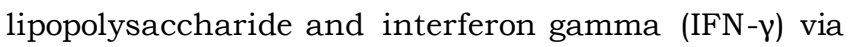
the NFkB and JAK/STAT1 pathways. These circumstances suggest that FGF-23 is produced by M1 macrophages and can modulate pro-inflammatory function in vitro and may support the innate immune response to tissue injury. Therefore, it is possible that endogenous expression and secretion of FGF-23 by infiltrating macrophages will induce the development of cardiovascular and renal disease through the induction of inflammation and fibrosis. 25,33

\section{Conclusion}

Elevated cardiac FGF23 is implicated in various clinical conditions such as CKD, ischemic cardiomyopathy, myocarditis, dilated cardiomyopathy, $\mathrm{HF}$ inflammation and ischemic reperfusion. At the cellular level FGF-23 is expressed in cardiac myocytes and non-cardiac myocytes such as cardiac fibroblasts, vascular smooth muscle, coronary artery endothelial cells and in macrophages. Based on the broad role of FGF-23, it is likely that FGF-23 is involved in the pathogenesis of CSFP.

\section{References}

1. Beltrame JF. Defining the coronary slow flow phenomenon. Circ J 2012; 76 : 818-820.

2. Wang $X$, Ping Nie S. The coronary slow flow phenomenon: characteristics, mechanisms and implications. Cardiovasc Diagn Ther 2011; 1 (1): 37-43

3. Separham A, Sohrabi B, et al. Clinical and laboratory predictors of coronary slow flow in coronary angiography. Perfusion 2017, Vol.32(1): 13-19

4. Ghanie A, Indrajaya $T$, Ali $Z$, Partan RU, dan Saleh MI. Molecular diagnostic marker, "Ace Polymorpgism Gen" in patient of slow coronary flow syndrome in Rumah Sakit Moehammad Hoesin Palembang. ACTAMedica 2017; 1-7

5. Asowata EO, Ilenwabor BP, Ebeigbe AB. Fibroblast growth factor-23: a potential cause of cardiovascular disease in chronic kidney disease patients. J. Afr. Ass. Physiol. Sci. July 2016; 4 (1): 1-6

6. Xiao Y, Peng C, Huang W, Zhang J, Xia M, et al. Circulating fibroblast growth factor 23 is associated with angiographic severity and extent of coronary artery disease. Plos One 2013; 8 (8): e72545

7. Sanghvi S, Mathur R, Baroopal A, Kumar A. Clinical, demographic, risk factor and angiographic profile of coronary slow flow phenomenon: A single centre experience. Indian Heart Journal 70 (2018) S290-S294

8. Gibson CM, Cannon CP, Daley WL, Dodge JT Jr, Alexander B Jr, Marble SJ, et al. TIMI frame count: A quantitative method of assessing coronary artery flow. Circulation 1996; 93: 879 $-888$

9. Zhu X, Shen H, Gao F, Wu S, Ma Q, et al. Clinical profile and outcome in patients with coronary slow flow phenomenon. Hindawi 2019; $2019: 1-7$

10. Park KH, Park WJ. Endothelial dysfunction: clinical implications in cardiovascular disease and therapeutic approaches. Journal of Korean Medical science 2015; 30 (9): 12131225

11. Sezgin N, Barutcu I, Sezgin AT, Gullu H, Turkmen M, Esen AM,et al. Plasma nitric oxide level and its role in slow coronary flow phenomenon. International Heart Journal 2005; 46 (3): 373-382

12. Nathani S. Role of endothelial function in coronary slow flow phenomenon with angiographically normal coronaries. Journal of Dr. NTR University of Health Sciences 2016; 21 (2): 146-155

13. Cin VG, Pakdemir H, Camsari. Diffuse intimal thickening of coronary arteries in slow flow coronary. Jpn Heart J 2003; 941-100 
14. Pakdemir H, Polat G, Cin VG, et al. Elevated plasma endothelin-1 levels in coronary sinus during rapid right atrial pacing in patients with slow coronary flow. Int J Cardio 2004, 97: 3541

15. Signory LU, Quadros ASD, Sbruzzi G, Dipp T, Lopes RD, Schaan BDA. Endothelial function in patients with slow coronary flow and normal coronary angiography. Clinics 2012; 67 (6): 677-680

16. Ramaswamy SD, Vigmostad SC, Wahle A, et al. Fluid dynamic analysis in a human left anterior descending coronary artery with arterial motion. Ann Biomed Eng 2004; 32: 1628-41

17. Chatzizisis YS, Coskun AU, Jonas M, et al. Role of endothelial shear stress in the natural history of coronary atherosclerosis and vascular remodeling: molecular, cellular, and vascular behavior. J Am Coll Cardiol 2007; 49: 2379-93

18. Katarci M, Gundogdu F, Doganay S, et al. Arterial bending angle and wall morphology correlate with slow coronary flow: determination with multidetector CT coronary angiography. Eur J Radiol 2011; 77: 111-7

19. Karakaya O, Kocer A, Esen AM, et al. Impaired cerebral circulation in patients with slow coronary flow. Tohuko J Exp Med 2011; 225: 13-6

20. Camsari A, Ozcan T, Ozer C, et al. Carotid artery intima-media thickness correlates with intravascular ultrasound parameters in patiens with slow coronary flow. Atherosclerosis 2008; 200: 310-4

21. Arat N, Altay H, Sabah I. Elastic properties of aorta are impaired in patients with slow flow coronary phenomenon. Indian Heart J 2008; 200:310-4

22. Wang X, Geng LL, Nie SP. Coronary slow flow phenomenon: a local or systemic disease? Med Hypotheses 2010; 75: 334-7

23. Vaidya A, Brown JM, Williams JS. The renin angiotensin aldosterone system and calcium regulatory hormones. J Hum Hypertens. Sept 2015; 29 (9): 515-521

24. Heras MM, Rodriguez NC, Gonzales JFN. The renin angiotensin aldosterone system in renal and cardiovascular disease and the effects of its pharmacological blockade. J Diabetes Metab 2012; 3 (1): 1-24

25. Nestler ML, Haffner D. Paracrine Effects of FGF-23 on the heart. Front. Endocrinol. May 2018; 9 (278); 86-96

26. Naing Z, Qiu CG, Han ZY. Endothelial dysfunction and inflammation: band master of the orchestra of slow coronary flow phenomenon. Journal of American College of Cardiology. April 2014; 63 (12): 1

27. Itoh N, Ornitz DM. Fibroblast growth factors: from molecular evolution to roles in development, metabolism and disease. J Biochem 2011; 149: 121-30

28. Brewer JR, Mazot P, Soriano P. Genetics insights into the mechanisms of Fgf signaling. Genes Dev 2016; 30: 751-71

29. Itoh N, Ohta H, Nakayama Y, Konishi M. Roles of Fgf signals in heart development, health, and disease. Front Cell Dev Biol 2016; 4: 110

30. Fon Tacer K, Bookout AI, Ding X, Kurosu H, John GB et al. Research resource: comprehensive expression atlas of the fibroblast growth factor system in adult mouse. Mol Endocrinol 2010; 24: 2010-0142

31. Saito T, Fukumoto S. Fibroblast growth factor 23 (FGF23) and disorders of phosphate metabolism. International Journal of Pediatric Endocrinology 2009: 1-6

32. Shimada T, Hasegawa H, Yamazaki Y, Muto T, Hino $\mathrm{R}$, et al. FGF-23 is a potent regulator of vitamin $\mathrm{D}$ metabolism and phosphate homeostasis. J Bone Miner Res 2004; 19: 42935

33. Haad CR, Santamaria R, Castaneda JRM, Ruiz de Mier MVD, Malo AM, et al. FGF23, biomarker or target. Toxin 2017; 11: 175

34. Tagliabracci VS, Engel JL, Wiley SE, Xiao J, Gonzales DJ, et al. Dynamic regulation of FGF- 
23 by fam20Cphosphorylation, GalNAc-T3 glycosilation and furin proteolysis. Proc Natl Acad Sci USA 2014; 111: 5520-5

35. Nestler ML, Grabner A, Hermann L, Richter B, Schmitz K, et al. Vitamin D treatment attenuates cardiac fgf-23/fgfr-4 signaling and hypertrophy in uremic rats. Nephrol Dial Transplant 2017; 32: 1493-503

36. Bienaime F, Ambolet A, Aussilhou B, Brazier F, Fouchard M, et al. Hepatic production of fibroblast growth factor-23 in autosomal dominant polycystic kidney disease. J Clin Endocrinol Metab 2018.

37. Sugiura H, Matshusita A, Futaya M, Teraoka A, Akiyama KI, et al. Fibroblast growth factor23 is upregulated in the kidney in a chronic kidney disease rat model. Plos One 2018; 13: e0191706

38. Han X, Yang J, Li I, Huang J, King G, et al. Conditional deletion of Fgfr 1 in the proximal and distal tubule identifies distinct roles in phosphate and calcium transport. Plos One 2016; 11: e0147845

39. Olausan H, Vervloet MG, Cozzolino M, Massy ZA, Torres PU, et al. New insight into the fgf23klotho axis. Seminars in Nephrology Nov 2014; (34)6: 586-597

40. Ornitz DM, Itoh N. The fibroblast signaling pathway. Wiley Interdiscip Rev Dev Biol 2015; 4: 215-66

41. Vogt I, Haffner D, Nestler ML. FGF23 and phosphate-cardiovascular toxins in CKD. Toxins 2019: (11)647: 1-23Erben RG. Physiological actions of fibroblast growth factor-23. Front. Endocrinol 2018; 9: 267

42. Erben RG. Physiological actions of fibroblast growth factor-23. Front. Endocrinol 2018; 9: 267

43. Richter B, Faul C. Fgf23 actions on target tissues-with and without klotho. Front. Endocrinol 2018; 9: 189

44. Akiyama KC, Miura Y, Hayashi H, Sakata A, Matsumura Y, et al. Calciprotein particles regulate fibroblast growth factor-23 expression in osteoblast. Kidney International 2019; 9: 111

45. Andrukova O, Streicher C, Zeith U, Erben RG. Fgf23 and parathyroid hormone signaling interact in the kidney and bone. Mol Cell Endocrinol 2016; 436: 224-39

46. Murali SK, Roschger P, Zeitz U, Klaushofer K, Andrukhova $\mathrm{O}$, et al. FGF23 regulates bone mineralization in a $1,25(\mathrm{OH}) \mathrm{D}$ and klothoindependent manner. J Bone Miner Res 2016; 31: $129-42$

47. Shalhoub V, Ward SC, Sun B, Stevens J, Renshaw I, et al. Fibroblast growth factor 23 (FGF23) and alpha-klotho stimulate osteoblastic MC3T3.E1 cell proliferation and inhibit mineralization. Calcif Tissue Int 2011; 89: $140-50$

48. Lutsey PL, Alonso A, Selvin E, et al. Fibroblast growth factor-23 and incident coronary heart disease,heart failure, and cardiovascular mortality: the Atherosclerosis Risk in Communities study. J Am Heart Assoc 2014; 3: e000936

49. Faul C, Amaral AP, Oskouei B, Hu MC, Sloan $A$, et al. FGF23 induces left ventricular hypertrophy. J Clin Invest 2011; 121: 43934408

50. Touchberry CD, Green TM, Tchikrizov V, Mannix JE, Mao TF, et al. FGF 23 is a novel regulator of intracellular calcium and cardiac contractility in addition to cardiac hypertrophy. American Journal of physiology Endocrinology and Metabolism 2013; 304 (8): E863-E873

51. Six I, Okazaki H, Gross P, Cagnard J, Boudot $\mathrm{C}$, et al. Direct acute effects of klotho and FGF23 on vascular smooth muscle and endothelium. Plos One April 2014; 9 (4): 1-9

52. Kumar S, Bogle R, Banerjee. Why do young people with chronic kidney disease die early? World J Nephrol November 2014; 3 (4): 143-155

53. Verkaik M, Juni RP, Loon EP, Poelgeest EM, Kwekkeboom RF, et al. FGF23 impairs peripheral microvascular function in renal 
failure. Am J Physiol Heart Circ Physiol 2018;

315: H1414-H1424 